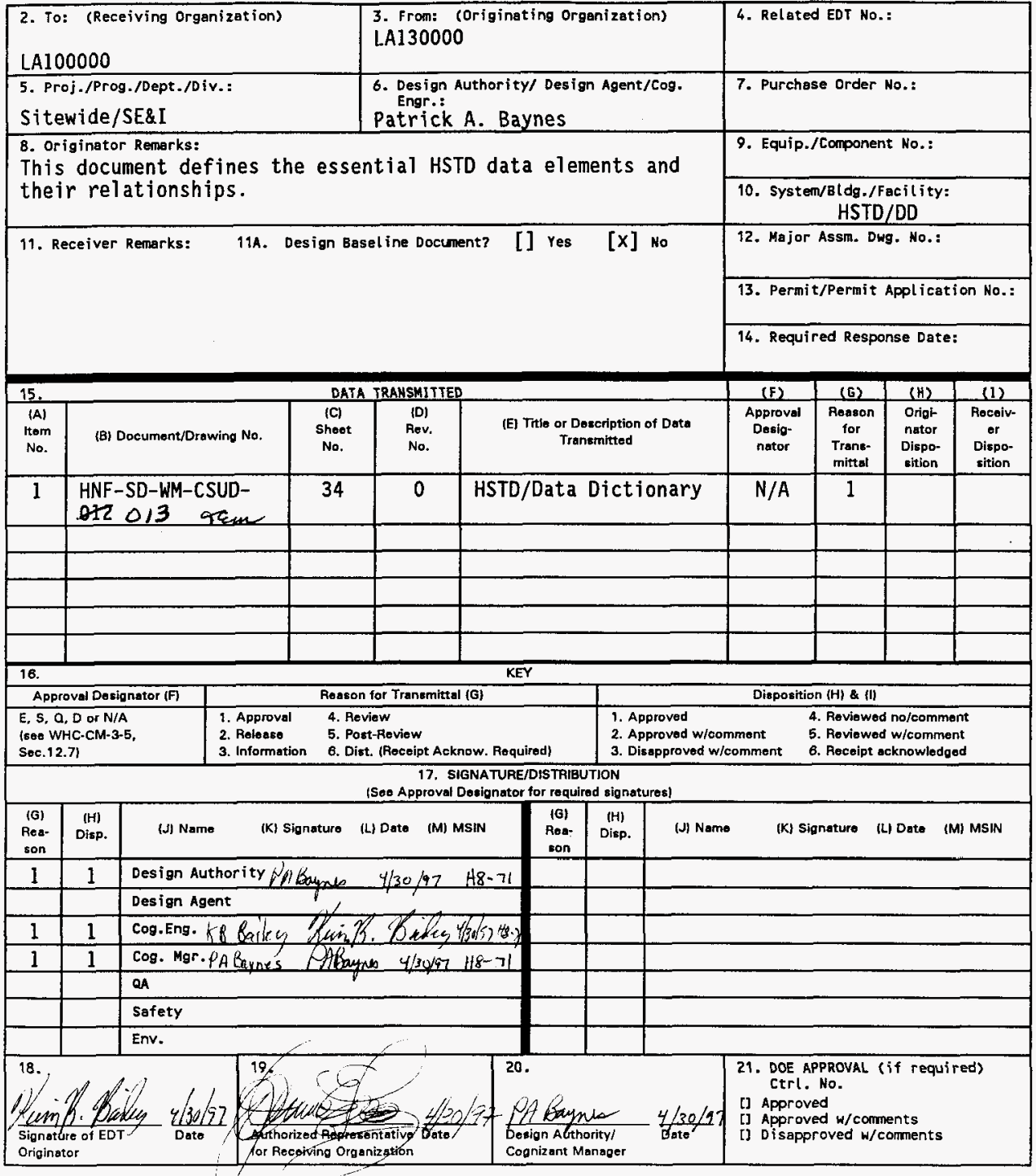

BD-7400-172-2 (05/96) GEFOOH 


\title{
Hanford Site Technical Baseline Data Dictionary
}

\author{
Patrick A. Baynes \\ Lockheed Martin Hanford Corporation, Richland, WA 99352 \\ U.S. Department of Energy Contract DE-AC06-96RL13200
}

\begin{tabular}{|c|c|c|}
\hline $\begin{array}{l}\text { EDT/ECN: } \\
\text { Org Code: } \\
\text { B\&R Code: }\end{array}$ & $\begin{array}{l}614851 \\
76130 \\
\text { EW3110010 }\end{array}$ & $\begin{array}{l}\text { UC: } 2080 \\
\text { Charge Code: } \\
\text { Total Pages: }\end{array}$ \\
\hline
\end{tabular}

Key Words: HSTD, Data Dictionary, Data Elements, Relationships

Abstract: The HSTD is the repository for Hanford site technical baseline data. It is used by Site System Engineering as the primary SE tool. This document defines the essential (required by RLID 430) HSTD data elements and their relationships.

This material was prepared as an account of work eponsored by an agency of the United States Government. Neither the United States Government nor the United States Department of Energy, nor any of their employees, nor any of their contractors, makes any warranty, express or implied, or assumes any legal liability or responalibilty for the scouracy, completenese, or usefulnese of any information, apparatus, product, or process disclosed, or represente that ite uee would not infringe privately owned rights. Reference to any specific commercial product, process or services by trede name, trademark, menufecturer, or otherwise does not necessarily constitute or imply its ondorsement, recommendation, or favoring by the United Stes Gavernment, lte agencies, nor employees, nor its contractors acting in its behalf.

TRADEMARK DISCLAIMER. Reference herein to any specific comercial product, process, or service by trade name, trademark, manufacturer, or otherwise, does not necessarily constitute or imply its endorsement, recommendation, or favoring by the United states Government or any agency thereof or its contractors or subcontractors.

Printed in the United States of America. To obtain copies of this document, contact: WHC/BCS Document Control Services, P.0. Box 1970, Mailstop M6-08, Richland WA 99352, Phone (5092 372-2420: Fax (509) $376-4989$.
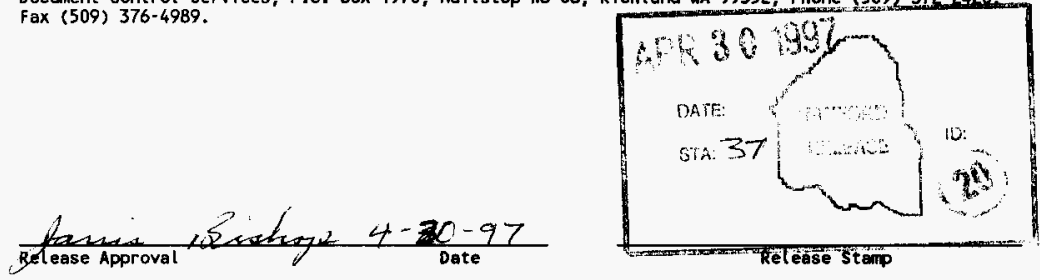
HNF-SD-WM-CSUD-013，Rev. 0

TABLE OF CONTENTS

1.0 INTRODUCTION . . . . . . . . . . . . . . . . . . . . . I

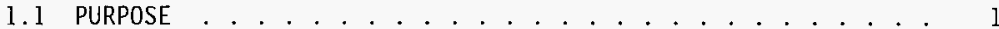

$1.2 \mathrm{SCOPE} \ldots \ldots \ldots \ldots \ldots$

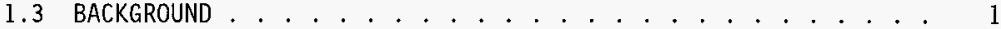

1.4 APPLICABILITY . . . . . . . . . . . . . . . . . . . 1

1.5 PREREQUISITES . . . . . . . . . . . . . . . 2

1.6 REFERENCE DOCUMENTATION . . . . . . . . . . . . . . 2

1.7 FONTS AND CONVENTIONS . . . . . . . . . . . . . . . 2

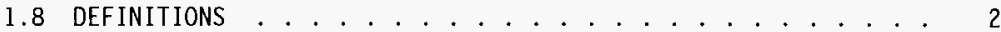

1.9 PRIMARY STRUCTURE OF THE HANFORD SITE TECHNICAL BASELINE DATABASE (HSTD) ................... 3

2.0 ELEMENTS, RELATIONSHIPS, AND ATTRIBUTES . . . . . . . . . . . 4

2.1 COMMON ATTRIBUTES . . . . . . . . . . . . . . . . . . . . . . 4

2.2 COMMON RELATIONSHIPS ................... 4

2.3 UNIQUE ELEMENTS, ATTRIBUTES, . . . . . . . . . . . . . 5

2.3.1 Category . . . . . . . . . . . . . . . 5

2.3 .2 Comment ............... . . . . 6

2.3 .3 Component ................. . . 6

2.3 .4 Criticallssue . . . . . . . . . . . . . 8

2.3 .5 Decision............. . . . . 9

2.3 .6 DiscreteFunction ............. . 11

2.3 .7 DiscreteItem . . . . . . . . . . . . . . 12

2.3.8 DVFChartDescription ............ . . . 13

2.3.9 DVFTimeL ineDescription . . . . . . . . . . . 14

2.3.10 EventForecast . . . . . . . . . . . 15

2.3.11 InfrastructureForecast ............ 16

2.3 .12 Interface . . . . . . . . . . . . . . 17

2.3 .13 ItemLink . . . . . . . . . . . . . . . 18

2.3.14 MaterialForecast . . . . . . . . . . . . 19

2.3 .15 Organization . . . . . . . . . . . . 21

2.3 .16 Scenario . . . . . . . . . . . . 22

2.3 .17 Source ................. . 23

2.3 .18 SystemRequirement . . . . . . . . . . . 24

2.3 .19 Timefunction ................ . 26

2.3 .20 Timeltem . . . . . . . . . . . . . . . . . 27

2.3 .21 WasteForecast . . . . . . . . . . . 28

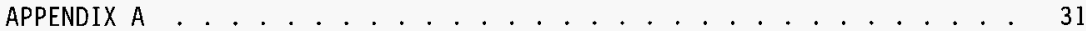


HNF-SD-WM-CSUD-013， Rev , 0

\section{HANFORD SITE TECHNICAL BASELINE DATABASE DATA DICTIONARY}

\subsection{INTRODUCTION}

\subsection{PURPOSE}

The purpose of this Data Dictionary is to provide information concerning the structure and information contained in the Hanford Site Technical Baseline Database (HSTD).

\subsection{SCOPE}

This Data Dictionary addresses the data elements, the attributes of each data element, and the relationships between data elements that are utilized within the RDD-100 ${ }^{\text {s }}$ systems engineering tool to describe and document the HSTD. The mechanics of the use of the RDD $100^{\circledR}$ tool to develop the database and to run reports on the database are covered in the user manual that comes with each copy of RDD-100@ and are not within the scope of this document.

\subsection{BACKGROUND}

The RDD-100 ${ }^{\circledR}$ suite of software tools support a structured systems engineering approach to developing complex systems. The RDD-100 tool suite is being used at Hanford to manage the site's and projects' technical baselines, including supporting data. The tool implements a object-oriented data structure in which data is represented as data elements (E), relationships ( $R$ ) between data elements, and attributes $(A)$ associated with each data element. Understanding of this ERA structure forms the basis for understanding the information contained in this document. The ERAs contained in this document are a subset of the schema ERAs available within the RDD-100 ${ }^{\circledR}$ tool (see reference $1.6 \mathrm{~b}$ ). The software requirements for the purchase of RDD-100 are documented in the software requirements specification (HNF-SD-WM-SDR-014).

\subsection{APPLICABILITY}

Information contained in this Data Dictionary is applicable to the HSTD as maintained by the Systems Engineering and Integration organization. Data submitted for inclusion into the HSTD will be checked for compliance with the mandatory attributes and relationships described in this document prior to being incorporated into a revised HSTD.

${ }_{\mathrm{RDD}-100}$ is a registered trademark of Ascent Logic Corporation 
HNF-SD-WM-CSUD-013， Rev. 0

\subsection{PREREQUISITES}

The user should have a comfortable grasp of the Element / Relationship / Attribute concept that underlies RDD-100 have at least the level of knowledge that the "Introduction to ROD-100®" training from Ascent Logic provides.

\subsection{REFERENCE DOCUMENTATION}

a) RDD-100 Users Guide, Ascent Logic Corporation

b) Schema Reference, Ascent Logic Corporation

c) Printout of the Schema Description Report generated from the Hanford Site Technical Baseline

d) Technical Issues Management List, HNF-MD-030

\subsection{FONTS AND CONVENTIONS}

The text in this document uses the following conventions: element types are in italics (e.g., Component); relation names are in lower case bold (e.g., documented by), and attributes are upper/lower case (e.g., Description). Mandatory attributes and relationships are underlined (e.g., Description, and documented by). Specific instances of elements or values of attributes are in quotation marks ( e.g., the TimeFunction "Pump Liquid Waste" has as a Duration "10.3"). The data type of a given attribute is enclosed in square brackets (e.g., The attribute Description is of type [string]).

\subsection{DEFINITIONS}

ALC

Attribute

Behavioral

Requirements

Component

DVF
Ascent Logic Corporation, the developers of RDD-100@.

Attributes describe objects that are represented by elements. Examples of attributes are Name, Description, Size, Number, Maximum Value, Minimum Value, and Units.

Requirements that influence the manner in which the system behaves. A system's behavior is defined by what the system does and how well the system performs what it does. Behavioral requirements are then either functional or performance requirements and are linked to the system functions, which, in-turn, are linked to the architectures performing the functions.

The hardware and software required to implement the functionality contained in the TimeFunction(s) and DiscreteFunction(s), e.g., a subsystem, system, or major facility.

Dynamic Verification Facility - The modeling capability of RDD-100. 
HNF-SD-WM-CSUD-013， Rev. 0

Element

Element

Instance

FNet

Instance

Leaf Level

Non-behavioral

Requirements

Performance

Requirements

Relationship

Schema
Objects contained in the HSTD used to describe the Hanford Environmental Management System, e.g. TimeFunction, TimeItem, Component, SystemRequirement, Critical Issue, Decision, and Engineer.

An occurrence of a specific element type in the HSTD.

An automatically generated representation of the decomposition of a TimeFunction or Scenario. Since it is automatically generated by RDD-100 this element will not be addressed in this document, but their relationships to the other elements contained in this document are depicted in Appendix A.

An occurrence of a specific element, relationship, or attribute.

In hierarchical element types, a leaf level element is an element which is not broken into component parts.

Requirements that apply to the system architecture selected to perform the functions. Non-behavioral requirements are linked directly to the system architectures to which they apply. Environmental, design, and construction requirements are examples of non-behavioral requirements.

Requirements that indicate how well a function must be performed.

Relationships describe the role of each element relative to other elements. Relationships are a two-way link, and therefore, provide forward and backward traceability from either element in the relationship. Examples of relationships are incorporates, incorporated by, owns, owned by, documents, and documented by.

A collection of ERAs available for use in the HSTD. For a given available element this would include the elements, attributes, and relations as defined in this Data Dictionary, as we1l as others that may be available but not used.

\subsection{PRIMARY STRUCTURE OF THE HANFORD SITE TECHNICAL BASELINE DATABASE (HSTD)}

A graphical depiction of the primary structure (main elements and relationships) of the HSTD is contained in Appendix A. The appendix addresses the elements and relationships utilized to build the Hanford Environmental Management System, along with the elements of a scenario (specifically the Life Cycle Asset Management [LCAM] scenario) and their relationship to the Hanford Cleanup System elements. The appendix also separately addresses the elements and relationships associated with appending of comments, categorization of elements, traceability of requirements, issue tracking and 
resolution, and establishment of hierarchical and organizational relationships. These are addressed separately since they are situations that may exist and therefore have a relationship with almost any other element of the HSTD.

Appendix A addresses the primary structure of the HSTD only. Other elements, relationships, and associated attributes are available within the HSTD as implemented in the RDD-100@ systems engineering tool. These ERAs are described in the reference documentation 1 isted above. The primary ERAs are further described in Section 2.0 of this document.

\subsection{ELEMENTS, RELATIONSHIPS, AND ATTRIBUTES}

2.1 COMMON ATTRIBUTES. Al1 element types in RDD-100@ have the following common attributes:

Author: [symbol] When an element is created, RDD-100® automatically fills this in using the contents of the Author field under User Preferences. This attribute documents the originator of an element instance. This can be user modified in the same manner as any other attribute. For example, if an engineer creates a Critical Issue for a manager who does not have access to RDD-100®, the engineer would overwrite the provided name with the manager's name.

Creation Date: [date] When an element is created, RDD-100 ${ }^{\circledR}$ automatically fills this in using the current date.

Modification Date: [date] When an element is created or changed in any way, RDD-100 automatically fills this in using the current date.

Modification Time: [time] When an e]ement is created or changed in any way, RDD-100® automatically fills this in using the current time.

Name: [string] Provides the element with an identifier which must be unique within its element type. An element is created when its name is defined.

2.2 COMMON RELATIONSHIPS. All elements in RDD-100® have the following relationships:

viewed by an RDD-100 diagram view. Identifies all of the types of views that the element has been shown as.

currently viewed by an $R D D-100^{\circ}$ diagram view. Identifies the most recent view (i.e., IDEFO, custom hierarchy) that the element has been shown as.

owned by Engineer. Identifies the owner of an element. RDD-100巴 automatically makes this relationship when an element instance is first created. The owner is the name of the Engineer in the Author field under User Preferences set by the user. This may be an individual's name or a more global designation such as "Site Systems Engineering," "TWRS Systems Engineering," etc. The element type Engineer can be 
HNF-SD-WM-CSUD-013， Rev. 0

found in the System Administration facility. This relationship is used as a part of the change control process.

\subsection{UNIQUE ELEMENTS, ATTRIBUTES, AND RELATIONSHIPS}

\subsubsection{Category}

\section{Definition/Usage}

Categories are a group or set of data elements that share common characteristics. Categorization of information and data is used as a means of placing element instances in groups for ease of analysis and reporting.

\section{ATTRIBUTES}

Category elements have the common attributes plus the following:

Category Element: [enumerated] "nil" (default), "Component," "CriticalIssue," "Decision," "Discretefunction," "Discreteltem", "Organization," "SystemRequirement, " "Scenario," "Source,"

"TimeFunction," "TimeItem," "VerificationRequirement." Used to indicate that this instance of Category is intended to be applied only to the chosen element type.

Description: [string] Used to describe what the link to this element is meant to signify.

Number: [hierarchical number] Used for sorting. Numbers are assigned in a hierarchical order, so that the first subelement to an element numbered $x . y . z$ would be numbered $x . y . z .1$, the second $x . y . z .2$ and so on.

Title: [string] The title of the category contained in the element instance.

\section{RELATIONSHIPS}

Category elements have the common relationships, plus the following:

annotated by Comment. Used to associate any non-requirement, non-issue text with an element.

categorized by Category. Used to group elements.

categorizes Any element.

incorporated by Category. Allows for the building of a hierarchy of Category elements. For example, lower level instances such as "Rail Transport," "Truck Transport," and "Ship Transport" are incorporated by a high level Category "Transportation."

incorporates Category. Allows for the building of a hierarchy of Category elements. For example, a high level Category "Transportation" incorporates lower level instances such as "Rail Transport," "Truck Transport," and "Ship Transport." 
HNF-SD-WM-CSUD-013，Rev. 0

\subsubsection{Comment}

Definition/Usage

Comments are textual information the user wants to relate to element instance(s). The difference between a comment and a criticallssue is: a Criticallssue requires action (i.e., it must be dealt with and resolved by a Decision) and a Comment does not.

\section{ATTRIBUTES}

Comment elements have the common attributes plus the following:

Description: [string] Used to describe what the link to this element is meant to signify.

Number: [hierarchical number] Used for sorting. Numbers are assigned in a hierarchical order, so that the first subelement to an element numbered $x . y . z$ would be numbered $x . y . z .1$, the second $x . y . z .2$ and so on.

Title: [string] The title of the element instance.

\section{RELATIONSHIPS}

Comment elements have the common relationships, plus the following:

annotates Any e7ement.

annotated by Comment. Used to associate any Comment with another Comment.

categorized by Category. Allows the user to group elements.

primary is Organization. Shows the Organization that has primary interest in the Comment.

secondary is Organization. Used to show the Organizations which have a secondary interest in the Comment.

\subsubsection{Component}

\section{Definition/Usage}

Component elements are used to accommodate the system architecture information. They are hierarchically structured through the built-in and built-from relation. Thus a Component can represent an entire system or any lower part of a system.

The interaction between Components is represented in the Component connects to Interface relation. The Interface is the collection point for all links joining a pair of Components (See Interface and ItemLink elements). Each Component connects to at least one Interface. Connected Components should be at the same level of the hierarchy. 
A Component is linked to the function it performs by the performs relation. Alternatively, the lowest level Component in the component hierarchy is 1 inked to the corresponding lowest level TimeFunction or DiscreteFunction in the functional hierarchy using the allocates relation.

The Component is used to capture the Hanford Site Environmental Management System hierarchy, including Major Facilities, e.g., "Plutonium Uranium Extraction (PUREX) Plant," "Plutonium Finishing Plant (PFP)," "Canister Storage Building (CSB)," "Reactors on the River Groundwater Operational Units," and "Central Plateau Soil Site Operational Units."

\section{ATTRIBUTES}

Component elements have the common attributes, plus the following:

Component Type: [enumerated] "nil," "CSC" (Computer Software Component), "CSCI" (Computer Software Configuration Item), "CSU" (Computer Software Unit), "Environment," "External System," "Facility," "Human," "HWCI" (Hardware Configuration Item), "HW element, " "Part," "Subsystem," "System," "System Segment," "Task."

Description: [string] Used to describe the component, system, subsystem, major facility, etc., represented by the element instance.

Number: [hierarchical number] Used for sorting. Numbers are assigned in a hierarchical order, so that the first subelement to an element numbered $x . y . z$ would be numbered $x . y . z .1$, the second $x . y . z .2$ and so on.

Title: [string] The title of the element instance.

\section{RELATIONSHIPS}

Component elements have the common relationships plus the following:

annotated by Comment. Used to associate any non-requirement, non-issue text with an element.

built from Component. Used to show how a Component is built from lower level Components.

built in Component. Used to show how a Component is built in its parent Component.

Note: Either built from or built in are mandatory, depending on hierarchy.

categorized by Category. Used to group elements.

connected to Interface. This relationship is used to show how one Component is connected to another via an Interface.

has context FNet The target FNet is used to include major facility into the life cycle Scenario of the Component. 


$$
\text { HNF-SD-WM-CSUD-013， Rev. O }
$$

inputs from ItemLink. This relation is used to link the destination Component to an ItemLink.

outputs to ItemLink. This relation is used to link the source Component to an ItemLink.

performs DiscreteFunction, TimeFunction. This relationship is used to link a Component to a TimeFunction that will represent the Component's behavior.

primary is Organization. Used to show the Organization which has responsibility for the Component.

raises Criticallssue. Used to indicate that someone has an issue with an aspect of the Component.

resulted from Decision. The target Decision is used to document a selection of architectural alternatives, and the alternative selected or the choice that resulted in an enabling assumption.

secondary is organization. Used to show the Organizations which have an involvement in the Component.

traced from SystemRequirement. The target systemRequirement is used to allocate non-behavioral requirements to the Component.

\subsubsection{Critical Issue}

\section{Definition/Usage}

Criticallssues are problems with the system design that must be resolved by a Decision. They are used to identify, track and provide traceability of conditions to be resolved. Any element within the schema may have a CriticalIssue attached. If the problem cannot be resolved without further analysis, the Description attribute must give a top-level explanation of the analysis that must take place. Each Criticallssue must have an Organization element to define the individual responsible to see that it is resolved.

\section{ATTRIBUTES}

Criticallssue elements have the common attributes plus the following:

Actual Date: [date] The date that the issue was finally resolved. The Actual Date attribute is mandatory when the issue is finally resolved.

Description: [string] The problem statement or the identification of a need for additional analysis.

Due Date: [date] The date the CriticalIssue was originally scheduled to be resolved.

Impact: [string] A list of items that will be affected by the resolution of the issue that are not already linked to the criticalIssue. 
HNF-SD-WM-CSUD-013， Rev . 0

Number: [hierarchical number] Used for sorting. Numbers are assigned in a hierarchical order, so that the first subelement to an element

numbered $x . y . z$ would be numbered $x . y . z .1$, the second $x . y . z .2$ and so on.

Priority: [enumerated] "A (Very High), "B (High), " "C (Medium), " D (Low)." (See reference $1.6 \mathrm{~d}$ ).

TitTe: [string] The title of the element instance.

\section{RELATIONSHIPS}

CriticalIssue elements have the common relationships plus the following:

annotated by Comment. Used to associate any non-requirement, non-issue text with an element.

categorized by Category. Used to group elements.

dependency of CriticalIssue. Used to show which Criticallssues cannot be resolved until the current Criticallssue has been resolved.

dependent on Criticallssue. Used to show the Criticallssues which must be resolved before the current Criticallssue may be resolved.

primary is Organization. The organization or person responsible to ensure the criticallssue is resolved.

raises Criticallssue. The target Criticallssue is used if the original criticallssue has a problem.

raised by Any element. Used to indicate the element with which a Criticalissue is associated.

resolved by Decision. The target Decision is used to capture the information that resolves the Criticalissue or results in an enabling assumption. The target relationship is mandatory when the issue is resolved or if an enabling assumption has been made.

secondary is Organization. Used to show the Organizations which have an interest in the outcome of the Criticallssue.

\subsubsection{Decision}

\section{Definition/Usage}

Decision elements are used to document the resolution of Criticallssues. The various attributes of the Decision element document the problem, a selection of alternatives, and the choice made.

For traceability, each Decision must be documented by a Source element that provides information concerning the source of the decision. 
HNF-SD-WM-CSUD-013， Rev. 0

\section{ATTRIBUTES}

Decision elements have the common attributes plus the following:

Alternatives: [string] The alternatives considered for resolving the problem; usually two to four alternatives are listed.

Choice: [string] Contains the alternative that was decided upon. The Choice attribute is mandatory at the time an alternative is selected or a decision is made.

Decision Vehicle: [string] Method or process used to reach the decision (e.g., Optimization Techniques, Simulation/Modeling, etc.).

Number: [hierarchical number] Used for sorting. Numbers are assigned in a hierarchical order, so that the first subelement to an element

numbered $x . y . z$ would be numbered $x . y . z .1$, the second $x . y . z .2$ and so on.

Problem: [string] An analysis of the problem resolved by this Decision.

Status: [enumeration] "nil" (default), "Open," "Enabling Assumption," "Resolved." Indicates whether the Decision is interim, final or needs to be dealt with. The Status attribute of a given Decision may change over time.

Title: [string] The title of the element instance.

\section{RELATIONSHIPS}

Decision elements have the common relationships plus the following:

annotated by Comment. Used to associate any non-requirement, non-issue text with an element.

categorized by Category. Used to group elements.

documented by Source. The document that the Decision used as a basis for the choice. A Decision must be documented by a Source if the Decision Status is set to "Enabling Assumption" or "Resolved".

primary is Organization. The organization or person responsible for making the decision. A Decision must have a primary is relationship to an Organization if the Decision Status is set to "Open" or "Enabling Assumption."

resolves CriticalIssue. Links the Decision to the Criticallssue which it was created to address.

results in $A 71$ Elements. Used to document the effect the decision or enabling assumption has on the HSTD e.g., the target component could be used to document a selection of architectural alternatives. The target SystemRequirement could be used to document the result of a Decision concerning the interpretation of a higher level systemRequirement. The target SystemRequirement could also be used to document the result of a 
Decision that is enabling assumption.

raises Criticallssue. Used when an issue has been raised with some aspect of the Decision.

secondary is Organization. Used to show the Organization(s) that are affected by the outcome of the Decision, but are not the decision maker.

\subsubsection{Discretefunction}

\section{Definition/Usage}

A Discretefunction is a function at the lowest level of interest. Unlike a TimeFunction, a Discretefunction can not be decomposed into Timefunctions or DiscreteFunctions.

\section{ATTRIBUTES}

Discretefunction elements have the common attributes plus the following:

Description: [string] The element description.

Number: [hierarchical number] Used for sorting. Numbers are assigned in a hierarchical order, so that the first subelement to an element numbered $x \cdot y \cdot z$ would be numbered $x \cdot y \cdot z \cdot 1$, the second $x \cdot y \cdot z \cdot 2$ and so on.

Title: [string] The title of the element instance.

\section{RELAT IONSHIPS}

Discretefunction elements have the common relationships plus the following:

allocated to Component. Defines the Component that performs the DiscreteFunction.

annotated by Comment. Used to associate any non-requirement, non-issue text with an element.

categorized by Category. Used to group elements.

inputs DiscreteItem. Allows the user to show the flows which the Discretefunction is receiving.

outputs DiscreteItem. Allows the user to show the flows for which the Discretefunction is a source.

performed by Component. This relationship is used when the System Specifications are being formulated. It links the Discretefunction to the Component that will perform the function.

primary is Organization. Use to designate the Organization that is primarily responsible for the element. 
HNF-SD-WM-CSUD-013， Rev. 0

raises Criticallssue. Used to show that someone has an issue with the Discretefunction.

referred by FNet. Identifies the FNet that the Function is on.

Maintained by RDD-100® ${ }^{\circledR}$ and cannot be modified by the user.

resulted from Decision. Used to show the Decision which caused the Discretefunction to be created.

secondary is Organization. Use to designate the Organization that has secondary responsibility for the element.

traced from SystemRequirement. The target SystemRequirement is used to link a functional requirement with its corresponding function.

\subsubsection{DiscreteItem}

\section{Definition/Usage}

DiscreteItems are used to represent the inputs and outputs of functional elements such as Scenarios, DiscreteFunctions and TimeFunctions. They are the lowest level of decomposition of a product and represent products such as information, waste streams, canisters, etc. Unlike TimeItems, DiscreteItems cannot be further decomposed.

\section{ATTRIBUTES}

Discreteltem elements have the common attributes, plus the following:

Data Type: [enumerated] "nil" (default), "Boolean, " "Float," "Integer," "String," "Text," "Iarray," "Farray," "Sarray," "Blab (undefined Abs)," "File (ASCII, bin, etc.)," "Record," or "Database."

Description: [string] The element description.

IDEF0 Type: [enumerated] "Input," "Control," "Mechanism." Specifies the type of IDEF0 element the DiscreteItem is intended to be.

Item Type: [enumerated] "nil" (default), "physical," "data," "digital," "event."

Number: [hierarchical number] Used for sorting. Numbers are assigned in a hierarchical order, so that the first subelement to an element numbered $x, y, z$ would be numbered $x, y, z .1$, the second $x . y . z .2$ and so on.

Title: [string] The title of the element instance.

\section{RELATIONSHIPS}

DiscreteItem elements have the common relationships, plus the following:

annotated by comment. Used to associate any non-requirement, non-issue text with an element. 
carried by ItemLink. Used to show which ItemLinks the current TimeItem flows through.

categorized by Category. Used to group elements.

forecasted by MaterialForecast, WasteForecast, InfrastructureForecast, EventForecast. Used to show the forecast for the product represented by the DiscreteItem.

illustrated by DVFChartDescription. Used to associate a graphical depiction contained in a DVFChartDescription, such as a waste profile, with its associated TimeItem.

input to Scenario, TimeFunction, DiscreteFunction. Used to show the functions to which the current TimeItem flows are an input.

output from Scenario, TimeFunction, Discretefunction. Used to show the functions from which the current TimeItem is an output.

primary is Organization. Use to designate the Organization that is primarily responsible for the element.

raises Criticalissue. Used to show that someone has an issue with the DiscreteItem.

resulted from Decision. Used when the current DiscreteItem was created as a result of the resolution of a Decision.

secondary is Organization. Use to designate the Organization that has secondary responsibility for the element.

traced from SystemRequirement. Used to associate Non-Behavioral requirements.

\subsubsection{DVFChartDescription}

\section{Definition/Usage}

DVFChartDescription is a data element that is utilized to capture the data points necessary to depict information in chart form.

\section{ATTRIBUTES}

DVFChartDescription elements have the common attributes plus the following:

Description: [string] The element description.

Number: [hierarchical number] Used for sorting. Numbers are assigned in a hierarchical order, so that the first subelement to an element numbered $x . y . z$ would be numbered $x . y . z .1$, the second $x . y . z .2$ and so on.

Raw Data Points: [ordered collection] Used to store the data points for generating a graph. 


$$
\text { HNF-SD-WM-CSUD-013， Rev. } 0
$$

Title: [string] The title of the element instance.

X Label: [string] Used to define the Tabel for the $X$ axis of a graph.

$Y$ Label: [string] Used to define the labe T for the $Y$ axis of a graph.

\section{RELATIONSHIPS}

DVFChartDescription elements have the common relationships plus the following:

annotated by Comment. Used to associate any non-requirement, non-issue text with an element.

categorized by Category. Used to group elements.

illustrates TimeItem or DiscreteItem Used to associate the DVFChartDescription with the target behavioral item.

\subsubsection{DVFTimeLineDescription}

\section{Definition/Usage}

DVFTimeLineDescription is a data element that is utilized to capture the time Tine information concerning the outputs of a Scenario.

\section{ATTRIBUTES}

DVFTimeLineDescription elements have the common attributes plus the following:

Description: [string] The element description.

Number: [hierarchical number] Used for sorting. Numbers are assigned in a hierarchical order, so that the first subelement to an element numbered $x, y . z$ would be numbered $x, y, z .1$, the second $x . y . z .2$ and so on.

Title: [string] The title of the element instance.

\section{RELATIONSHIPS}

DVFTimelineDescription elements have the common relationships plus the following:

annotated by Comment. Used to associate any non-requirement, non-issue text with an element.

categorized by Category. Used to group elements.

illustrates Scenario Used to associate the DVFTimeLineDescription with the target Scenario. 
HNF-SD-WM-CSUD-013， Rev . 0

\subsubsection{EventForecast}

\section{Definition/Usage}

EventForecast is a data element that is utilized to capture the estimated events for a specific behavior item. It contains event forecast information for a specific fiscal year.

\section{ATTRIBUTES}

Eventforecast elements have the common attributes plus the following:

Description: [string] The element description.

Number: [hierarchical number] Used for sorting. Numbers are assigned in a hierarchical order, so that the first subelement to an element numbered $x . y . z$ would be numbered $x . y . z, 1$, the second $x . y . z .2$ and so on.

Schedule Date: [date] Schedule date for the event.

Title: [string] The title of the element instance.

\section{RELATIONSHIPS}

EventForecast elements have the common relationships plus the following:

annotated by Comment. Used to associate any non-requirement, non-issue text with an element.

categorized by Category. Used to group elements.

documented by Source Allows the user to document the source of the information contained in the EventForecast.

forecasts DiscreteItem, TimeItem Allows the user to show the DiscreteItem or TimeItem for which the event is being forecasted.

primary is Organization. Shows the Organization which is responsible for the information contained in the EventForecast.

raises Criticallssue. Used to show that someone has an issue with the EventForecast.

resulted from Decision. Used to show the Decision which caused the EventForecast to be created.

secondary is Organization. Shows the Organization which has a secondary interest in the information contained in the EventForecast. 
HNF-SD-WM-CSUD-013， Rev. 0

\subsubsection{InfrastructureForecast}

\section{Definition/Usage}

Infrastructureforecast is a data element that is utilized to capture the estimated infrastructure requirements for a specific behavior item. It contains infrastructure forecast information for a specific fiscal year.

\section{ATTRIBUTES}

Infrastructureforecast elements have the common attributes plus the following:

Description: [string] The element description.

Fiscal Year: [Integer-4 digit] Fiscal year for which the InfrastructureForecast is being made, i.e., 1997.

Infrastructure Type: [enumerated 1ist] Type of infrastructure being forecasted; i.e., "Allocated Land," "Analytical Laboratory Services," "Bioassay and Dosimetry Services," "Biological Laboratory Services," "Building Maintenance," "Clean Laundry," "Custodial Services," "Data (HLAN) Transmission," "Development Laboratory Services," "Electricity," "Energy Management Services," "Environmental Molecular Science Lab Services," "Fabrication Shop Services," "Guaranteed Ride Home," "Hanford Road System Heavy Traffic," "Heavy Equipment," "Heavy Trucks," "In-Field Laboratory Services," "Industrial Hygiene Services," "Lifting (Cranes)," "Non-radiation Standards (Calibrations)," "0ffice Space (Leased)," "Office Space (Infrastructure Owned)," "Office Space (Program Owned)," "Pager Service," "Potable Water," "Radioactive Standards (Calibrations)," "Rail Transportation," "Raw Water," "Sedans/Light Trucks," "Steam," "Storage Space (Infrastructure Owned)," "Storage Space (Leased)," "Storage Space (Program Owned)," "Taxi Service," "Video Communications," and "Voice (Telephone) Communication."

Maximum Value: [Floating Point] Maximum value of infrastructure forecasted for the fiscal year.

Mean Value: [Floating Point] Mean value of infrastructure forecasted for the fiscal year.

Minimum Value: [Floating Point] Minimum value of infrastructure forecasted for the fiscal year.

Number: [hierarchica] number] Used for sorting. Numbers are assigned in a hierarchical order, so that the first subelement to an element numbered $x . y . z$ would be numbered $x . y . z .1$, the second $x . y . z .2$ and so on.

Title: [string] The title of the element instance.

Units: [text] Unit of measure for the forecasted infrastructure. 
HNF-SD-WM-CSUD-013， Rev . O

\section{RELATIONSHIPS}

InfrastructureForecast elements have the common relationships plus the following:

annotated by Comment. Used to associate any non-requirement, non-issue text with an element.

categorized by Category. Used to group elements.

documented by Source Allows the user to document the source of the information contained in the InfrastructureForecast.

forecasts DiscreteItem, TimeItem Allows the user to show the DiscreteItem or TimeItem for which the infrastructure is being forecasted.

primary is Organization. Shows the Organization which is responsible for the information contained in the InfrastructureForecast.

raises Criticallssue. Used to show that someone has an issue with the InfrastructureForecast.

resulted from Decision. Used to show the Decision which caused the Infrastructureforecast to be created.

secondary is Organization. Shows the Organization which has a secondary interest in the information contained in the

InfrastructureForecast.

\subsubsection{Interface}

\section{Definition/Usage}

An interface is where two or more Components meet and act on, or interact with, each other. Interfaces are used to define the physical connection between two or more components. Interfaces can be structured in a hierarchy in the same way as Components. An Interface at a given level of the Component hierarchy may be bundled into a higher level Interface at a higher level of the Component hierarchy.

\section{ATTRIBUTES}

Interface elements have the common attributes plus the following.

Description: [string] The description of the element instance.

Number: [hierarchical number] Used for sorting. Numbers are assigned in a hierarchical order, so that the first subelement to an element numbered $x, y . z$ would be numbered $x, y, z .1$, the second $x, y, z .2$ and so on.

Title: [string] The title of the element instance. 
HNF-SD-WM-CSUD-013， Rev. 0

\section{RELATIONSHIPS}

Interface elements have the common relationships, plus the following:

annotated by comment. Used to associate any non-requirement, non-issue text with an element.

built from Interface. Used to build a hierarchy of subordinate Interfaces.

built in Interface. Used to link to the parent Interface.

categorized by Category. Used to group elements.

connects to Component. Used to show which Components the current Interface is linked to.

contains ItemLink. Used to show the ItemLinks which are bundled into the current Interface.

primary is organization. The organization or person responsible for the Interface.

raises CriticalIssue. Used to show that someone has an issue with the Interface.

secondary is Organization. Used to show the Organization(s) other than the primary that are affected by the Interface.

traced from SystemRequirement. The target SystemRequirement is used to allocate non-behavioral requirements to the Interface.

\subsubsection{ItemLink}

\section{Definition/Usage}

The ItemLink element is used to represent a one-directional pathway for one or more TimeItems or DiscreteItems (inputs and outputs) to be carried from one Component to another. They represent the physical object that carries the items. For example, "Ten Tons of Soil" would be an instance of a DiscreteItem, "Full Dump Truck" would be an instance of an ItemLink.

\section{ATTRIBUTES}

ItemLink elements have the common attributes, plus the following:

Capacity: [integer] Indicates how many units of the item can be carried from one process to the other per unit of time.

Description: [string] The description of the element instance.

Is Constrained: [enumerated] "false" (default), "true." If set to true, the simulator will simulate the time for item flow based upon the item size and the capacity of the ItemLink. 
Number: [hierarchical number] Used for sorting. Numbers are assigned in a hierarchical order, so that the first subelement to an element numbered $x . y . z$ would be numbered $x, y, z .1$, the second $x . y . z .2$ and so on.

Title: [string] The title of the element instance.

\section{RELATIONSHIPS:}

ItemLink elements have the common relationships, plus the following:

annotated by comment. Used to associate any non-requirement, non-issue text with an element.

carries TimeItem, DiscreteItem. Used to show which behavioral items through the current ItemLink.

categorized by Category. Used to group elements.

input to Component. Used to show the Component that the ItemLink flows in to.

is contained by Interface. Shows the Interface which the ItemLink is aggregated into.

output to Component. Used to show the Component that the ItemLink flows out of.

primary is organization. The organization or person responsible for the It emLink.

secondary is Organization. Used to show the Organization(s) other than the primary that are affected by the ItemLink.

raises criticalissue. Shows that an issue has been raised on the current ItemLink.

resulted from Decision. The target Decision is used to document the Decision that resulted in the ItemLink.

traced from SystemRequirement. The target SystemRequirement is used to allocate behavioral requirements to the ItemLink.

\subsubsection{MaterialForecast}

\section{Definition/Usage}

Materialforecast is a data element that is utilized to capture the estimated material (i.e., non-waste) flow for a specific behavior item. It contains material forecast information for a specific fiscal year. 


\section{ATTRIBUTES}

Materialforecast elements have the common attributes plus the following:

Description: [string] The element description.

Fiscal Year: [Integer-4 digit] Fiscal year for which the material forecast is being made; i.e., 1997.

Forecast Type: [enumerated 1 ist] Type of material forecast; i.e., "Begin Inventory, " "Received," "Generated," "Reduced," "Transferred," "Disposed," and "End Inventory."

Material Type: [enumerated 1ist] Type of material being forecasted; i.e., "Cesium (Cs)," "Depleted Uranium (DU)," "Highly Enriched Uranium (HEU), "Low Enriched Uranium (LEU)," "Miscellaneous Special Nuclear Material," "Miscellaneous Nuclear Material," "Miscellaneous Nuclear Fuels, " "Natural Uranium (NU), " "Plutonium (Pu)," "Sodium (Na)," "Spent Nuclear Fuel (SNF)," and "Strontium (Sr)."

Maximum Value: [Floating Point] Maximum value of material forecasted for the fiscal year.

Mean Value: [Floating Point] Mean value of material forecasted for the fiscal year.

Minimum Value: [Floating Point] Minimum value of material forecasted for the fiscal year.

Number: [hierarchical number] Used for sorting. Numbers are assigned in a hierarchical order, so that the first subelement to an element numbered $x . y . z$ would be numbered $x . y . z .1$, the second $x . y . z .2$ and so on.

Title: [string] The title of the element instance.

Units: [text] Unit of measure for the forecasted material.

\section{RELATIONSHIPS}

Materialforecast elements have the common relationships plus the following:

annotated by Comment. Used to associate any non-requirement, non-issue text with an element.

categorized by Category. Used to group elements.

documented by Source Allows the user to document the source of the information contained in the Materialforecast.

forecasts DiscreteItem, TimeItem Allows the user to show the DiscreteItem or TimeItem for which the material is being forecasted.

primary is Organization. Shows the Organization which is responsible for the information contained in the Materialforecast. 
raises Criticalissue. Used to show that someone has an issue with the MaterialForecast.

resulted from Decision. Used to show the Decision which caused the Materialforecast to be created.

secondary is Organization. Shows the Organization which has a secondary interest in the information contained in the Materialforecast.

\subsubsection{Organization}

\section{Definition/Usage}

The Organization element identifies the project or individual that has primary responsibility for a given element. It is commonly used with Life Cycle Phase Scenarios to define the project having responsibility for executing a particular life cycle phase of a major facility (Component) or with Criticallssues and Decisions to assign responsibility for providing alternatives and solutions. The Organization elements that describe the Project Hanford Breakdown Structure (PHBS) are available within the HSTD for use in defining responsibility for accomplishment of other elements within the HSTD.

Any element instance can have only one Organization as primary responsibility, but can have many organization instances as secondary. Similar to Components, organizations form a hierarchy.

\section{ATTRIBUTES}

Organization elements have the common attributes plus the following:

Description: [string] The description of the element instance. The is attribute should be the mission statement when the organization element is used for a project and the individuals responsibility when the Organization element is used for a person.

Number: [hierarchical number] Used for sorting. Numbers are assigned in a hierarchical order, so that the first subelement to an element numbered $x . y . z$ would be numbered $x . y . z .1$, the second $x . y . z .2$ and so on.

Project Unique ID: [string] The project identifier used to uniquely identify each project within Project Hanford.

Title: [string] The title of the element instance.

\section{RELATIONSHIPS} following:

Organization elements have the common relationships, plus the

annotated by Comment. Used to associate any non-requirement, non-issue text with an element. 
built in Organization. This relationship allows the user to place Organization(s) in a hierarchy; i.e., lower level Organization(s) are built in to higher level Organization(s).

built from Organization. This relationship also allows the user to place Organization(s) in a hierarchy; i.e., higher level

Organization(s) are built from lower level Organization(s).

Note: Either built in or built from are mandatory depending on hierarchy.

categorized by Category. Used to group elements.

primary for $A 7 l$ elements. Used to assign responsibility for an element: With this relationship any given element instance can be linked to only one Organization (i.e., only one Organization and Responsible Individual has primary responsibility for a element instance).

secondary for $A 77$ elements. Used to define the people and organizations with secondary interest in the element. The organization that is primary for a given element instance should coordinate changes with the Organization(s) that are secondary for the same element instance.

Note: Either primary for or secondary for are mandatory.

\subsubsection{Scenario}

\section{Definition/Usage}

A Scenario is a function that can be decomposed into a sequence of other functions (i.e., TimeFunction, DiscreteFunction, or Scenarios). Scenarios are used to define a sequence of functions representing a single thread of operational behavior. Scenarios can be used to establish performance requirements.

Within the HSTD, the Scenario is used to develop the "Life Cycle Asset Management (LCAM)" scenario where each major facility is depicted as having a life cycle. The life cycle of each major facility is comprised of $1 \mathrm{ife}$ cycle phases of the facility components. These life cycle phases are also Scenarios and are comprised of the functions that must perform during that particular life cycle phase.

\section{ATTRIBUTES}

Scenario elements have the common attributes, plus the following:

Number: [hierarchical number] Used for sorting. Numbers are assigned in a hierarchical order, so that the first subelement to an element numbered $x . y . z$ would be numbered $x . y . z .1$, the second $x . y . z .2$ and so on. 
HNE-SD-WM-CSUD-013, Rev. 0

Description: [string] Provides the description for this instance of the element type.

Title: [string] The title of the element instance.

\section{RELATIONSHIPS}

Scenario elements have the common relationships, plus the following:

annotated by comment. Used to associate any non-requirement, non-issue text with an element.

categorized by Category. Used to group elements.

current decomposition of FNet. Identifies the FNet that currently decomposes the Scenario. [This is a one to one relationship.]

decomposed by FNet. Identifies all of the FNets that decompose the Scenario. [This is a one to many relationship.]

inputs DiscreteItem, TimeItem. Show the items which are flowing into the current Scenario.

illustrated by DVFTimeLineDescription Used to associated a graphical depiction contained in a DVFTimeLineDescription, with its associated Scenario.

outputs Discreteltem, TimeItem. Show the items which are flowing from the current Scenario.

primary is Organization. Used to assign responsibility for the Scenario, specifically the "LCAM Phases" scenarios.

raises Criticalissue. Used to show that someone has an issue with the Scenario.

referred by FNet. Identifies the FNet that the Scenario is on. Maintained by RDD- $100^{\mathrm{B}}$ and cannot be modified by the user.

secondary is organization. Used to define the people and organizations that need to remain cognizant of the Scenario.

\subsubsection{Source}

\section{Definition/Usage}

The Source element is used to provide traceability of information contained in a given element back to the source documentation. Its most common uses are as a starting point for a SystemRequirements hierarchy or as the basis of a Decision. 
HNF-SD-WM-CSUD-013， Rev . 0

\section{ATTRIBUTES}

Source elements have the common attributes plus the following:

Description: [string] This attribute stores descriptive text about the source of a requirement, decision, etc.

Number: [hierarchical number] Used for sorting. Numbers are assigned in a hierarchical order, so that the first subelement to an element numbered $x, y . z$ would be numbered $x, y, z .1$, the second $x, y, z .2$ and so on.

Source Type: [enumerated] "nil," "Originating Requirements, "Meeting Minutes," "Trade-off Study Report," "Change Request," "Project Memo," "Standard," "Other." "Originating Requirements" and "Standard" are" used as the starting point for an originating requirements hierarchy; "Trade-off Study Report" is used as the starting point for a derived or performance requirements hierarchy; "Meeting Minutes" and "Project Memo" are used as the starting point for Decisions.

Title: [string] The title of the document contained in the element instance.

\section{RELATIONSHIPS}

Source elements have the common relationships plus the following:

annotated by comment. Used to associate any non-requirement, non-issue text with an element.

categorized by Category. Used to group elements.

documents All element types. The most common targets for this relationship are Decisions, SystemRequirements, WasteForecast, MaterialForecast, InfrastructureForecast, and EventForecast.

invoked by SystemRequirement. This relation is used when a Source of requirements is identified in the body of another requirement (e.g., "...retrieval shall be in accordance with 10 CFR ...," then the requirement has invoked another Source).

raises Criticallssue. Used to show that someone has an issue with the Source.

\subsubsection{SystemRequirement}

The SystemRequirement element is used to capture the statements that identify the essentia? needs of the system under consideration: what the system is to do, how it is to be done, and the standards which must be met. SystemRequirements can come directly from source documents (e.g., DOE $5820.2 \mathrm{~A})$, or may be derived from analysis/trade studies.

Alt originating requirements must be documented by a Source. 
SystemRequirements form a hierarchy using the incorporates relationship. A11 SystemRequirements must either be documented by a Source or incorporated by a parent SystemRequirement. All leaf level Requirements must be linked to the appropriate element via the traces to relationship.

\section{ATTRIBUTES}

SystemRequirement elements have the common attributes plus the following:

Description: [string] The description of the element instance.

Number: [hierarchical number] Used for sorting. Numbers are assigned in a hierarchical order, so that the first subelement to an element numbered $x . y . z$ would be numbered $x . y . z .1$, the second $x . y . z .2$ and so on.

Title: [string] The title of the element instance.

\section{RELATIONSHIPS}

SystemRequirement elements have the common relationships plus the following:

annotated by Comment. Used to associate any non-requirement, non-issue text with an element.

categorized by Category. This relationship is used to place SystemRequirements into groups for ease of analysis.

documented by Source. This relationship allows the user to identify the original, verbatim Source of the text found in the SystemRequirement.

incorporated by SystemRequirement. This relationship allow the user to place SystemRequirements in a hierarchy (i.e., a lower level SystemRequirement is incorporated by higher level SystemRequirement). incorporates SystemRequirement. This relationship allow the user to place SystemRequirements in a hierarchy (i.e. a higher level SystemRequirement incorporates lower level SystemRequirements).

invokes Source. This relation is used when a systemRequirement identifies a source document as part of its requirement (e.g., "...retrieval shall be in accordance with 10 CFR ...").

raises Criticallssue. This relation is used to show that someone has an issue with an aspect of the SystemRequirement.

resulted from Decision. This relation is used to show that a SystemRequirement has been derived through the requirements management process. An incorporated by SystemRequirement relationship must accompany this relation.

traces to Component, DiscreteFunction, DiscreteItem, Interface, ItemLink, TimeFunction, TimeItem, (All other element types). TimeFunction, DiscreteFunction, and ItemLink are the targets for 
HNF-SD-WM-CSUD-013， Rev . 0

Behavioral requirements and Component and Interface are the targets for non-behavioral requirements. TimeItems and DiscreteItems are the targets for product specification requirements. A SystemRequirement must either incorporates another SystemRequirement or must have a traces to relationship to a valid target type.

\subsubsection{TimeFunction}

\section{Definition/Usage}

TimeFunctions are utilized to define what the system must do to meet its requirements and accomplish its mission. Within the HSTD, there are two types of functions, TimeFunctions and DiscreteFunctions. TimeFunctions may be further decomposed into lower level TimeFunctions or DiscreteFunctions. Discretefunctions are leaf nodes and therefore may not be further decomposed. All subfunctions, whether TimeFunctions or DiscreteFunctions, must contribute to performance of the higher level function. A Timefunction is the action performed by a Component.

\section{ATTRIBUTES}

Timefunctions have the common attributes plus the following:

Description: [string] The description of the element instance.

Number: [hierarchical number] Used for sorting. Numbers are assigned in a hierarchical order, so that the first subelement to an element numbered $x . y . z$ would be numbered $x . y . z .1$, the second $x . y . z .2$ and so on.

Title: [string] The title of the element instance.

\section{RELATIONSHIPS :}

TimeFunctions have the common relationships plus the following:

annotated by Comment. Used to associate any non-requirement, non-issue text with an element.

categorized by Category. Used to group elements.

current decomposition FNet. Identifies the FNet that currently decomposes the Function. [This is a one to one relationship.] This relationship is automatically created by RDD-100 ${ }^{8}$ when editing a Behavior Diagram.

decomposed by FNet. Identifies all of the FNets that decompose the Function, (i.e. the current decomposition and any alternate decompositions). [This is a one to many relationship.] This relationship is automatically created by $\mathrm{RDD}-100^{\circledR}$ when editing a Behavior Diagram.

inputs DiscreteItem, TimeItem. Show the items which are flowing into the current Timefunction. 
raises Criticallssue. Used when a user has an issue with some aspect of the current TimeFunction.

referred by FNet. Identifies the FNet that the TimeFunction is on, the parent function's FNet.

resulted from Decision. Used when the current TimeFunction is created as a result of resolving a Decision.

outputs DiscreteItem, TimeItem. Show the items which are flowing from the current TimeFunction.

performed by Component. Links a function with the Component that will perform the function. All leaf-level TimeFunctions must be performed by a Component.

primary is Organization. Use to designate the Organization that is primarily responsible for the element.

secondary is Organization. Use to designate the Organization that has secondary responsibility for the element.

traced from SystemRequirement. The target systemRequirement is used to allocate behavioral requirements to the function.

\subsubsection{TimeItem}

\section{Definition/Usage}

TimeItems are the inputs and outputs of functional elements such as Scenarios and Timefunctions. All items necessary for the function to perform are input to the function. Items transformed or produced by the function are output from the function. Timeltems can be decomposed into a hierarchy of lower level TimeItems or Discreteltems.

TimeItems of IDEFO Type "input" are the items or materials that enter a function and are transformed into products that are output from the function. TimeItems of IDEF0 Type "control" are management direction type of inputs that constrain the function. TimeItems of IDEF0 Type "mechan ism" are the items that are necessary for the function to transform the input material into an output product.

\section{ATTRIBUTES}

TimeItem elements have the common attributes plus the following:

Description: [string] The description of the element instance.

IDEF0 Type: [enumerated] Used to identify the type of TimeItem for use in developing the IDEFO diagrams. Available values are

"input"(default), "control," "mechanism."

Item Type: [enumerated] "nil" (default), "analog," "digital," "physical," "mixed." 
Number: [hierarchical number] Used for sorting. Numbers are assigned in a hierarchical order, so that the first subelement to an element numbered $x, y . z$ would be numbered $x . y, z .1$, the second $x . y . z .2$ and so on.

Title: [string] The title of the element instance.

\section{RELATIONSHIPS}

TimeItem elements have the common relationships plus the following:

annotated by comment. Used to associate any non-requirement, non-issue text with an element.

carried by ItemLink. Used to show which ItemLinks the current Timeltem flows through.

categorized by Category. Used to group elements.

forecasted by MaterialForecast, WasteForecast, InfrastructureForecast, or EventForecast. Used to show the forecast for the product represented by the TimeItem.

illustrated by DVFChartDescription. Used to associate a graphical depiction contained in a DVFChartDescription, such as a waste profile, with its associated DiscreteItem.

input to Scenario, TimeFunction, Discretefunction. Used to show the functions the current TimeItem flows into.

raises Criticallssue. Used when someone has an issue with the current TimeItem.

resulted from Decision. Used when the current TimeItem was created as a result of the resolution of a Decision.

output from Scenario, TimeFunction. Used to show the functions the current Timeltem flows out of.

primary is organization. Use to designate the Organization that is primarily responsible for the element.

secondary is Organization. Use to designate the Organization that has secondary responsibility for the element.

traced from SystemRequirement. Used to associate non-behavioral requirements.

\subsubsection{WasteForecast}

\section{Definition/Usage}

Wasteforecast is a data element that is utilized to capture the estimated waste flow for a specific behavior item. It contains volumetric waste forecast information for a specific fiscal year. 
HNF-SD-WM-CSUD-013， Rev. 0

\section{ATTRIBUTES}

Wasteforecast elements have the common attributes plus the following:

Description: [string] The element description.

Fiscal Year: [Integer-4 digit] Fiscal year for which the waste forecast is being made; i.e., 1997.

Forecast Type: [enumerated 1ist] Type of waste forecast; i.e., "Begin Inventory," "Received," "Generated," "Reduced, " "Transferred," "Disposed," and "End Inventory."

Maximum Value: [Floating Point] Maximum value of waste forecasted for the fiscal year.

Mean Value: [Floating Point] Mean value of waste forecasted for the fiscal year.

Minimum Value: [Floating Point] Minimum value of waste forecasted for the fiscal year.

Number: [hierarchical number] Used for sorting. Numbers are assigned in a hierarchical order, so that the first subelement to an element numbered $x . y . z$ would be numbered $x . y . z .1$, the second $x . y . z .2$ and so on.

Title: [string] The title of the element instance.

Units: [text] Unit of measure for the forecasted waste.

Waste Type: [enumerated 1ist] Type of waste being forecasted; i.e., "Asbestos," "CH LLMW I," "CH LLMW III," "CH LLW I," "CH LLW III, " $\mathrm{CH}$ TRU," "CH TRUM," "HAZ," "HLW," "Industrial Waste Water," "LLW (Liquid)," "LLMW (Liquid)," "RH LLMW GTCIII," "RH LLMW I, " "RH LLMW III," "RH LLW GTCIII," "RH LLW I," "RH LLW III," "RH TRU," "RH TRUM," "Sanitary Liquid Waste," "Sanitary Solid Waste," "Special Case Waste," and "Treated Liquid Effluent."

\section{RELATIONSHIPS}

Wasteforecast elements have the common relationships plus the following:

annotated by Comment. Used to associate any non-requirement, non-issue text with an element.

categorized by Category. Used to group elements.

documented by Source. Allaws the user to document the source of the information contained in the WasteForecast.

forecasts DiscreteItem, TimeItem. Allows the user to show the DiscreteItem or TimeItem for which the waste is being forecasted. 
primary is Organization. Shows the Organization which is responsible for the information contained in the WasteForecast.

raises Criticallssue. Used to show that someone has an issue with the Wasteforecast.

resulted from Decision. Used to show the Decision which caused the Wasteforecast to be created.

secondary is Organization. Shows the Organization which has a secondary interest in the information contained in the Wasteforecast. 
HNF-SD-WM-CSUD-013， Rev. 0

APPENDIX A

Hanford Site Technical Baseline Database Structure 
HNF-SD-WM-CSUD-013， Rev. 0

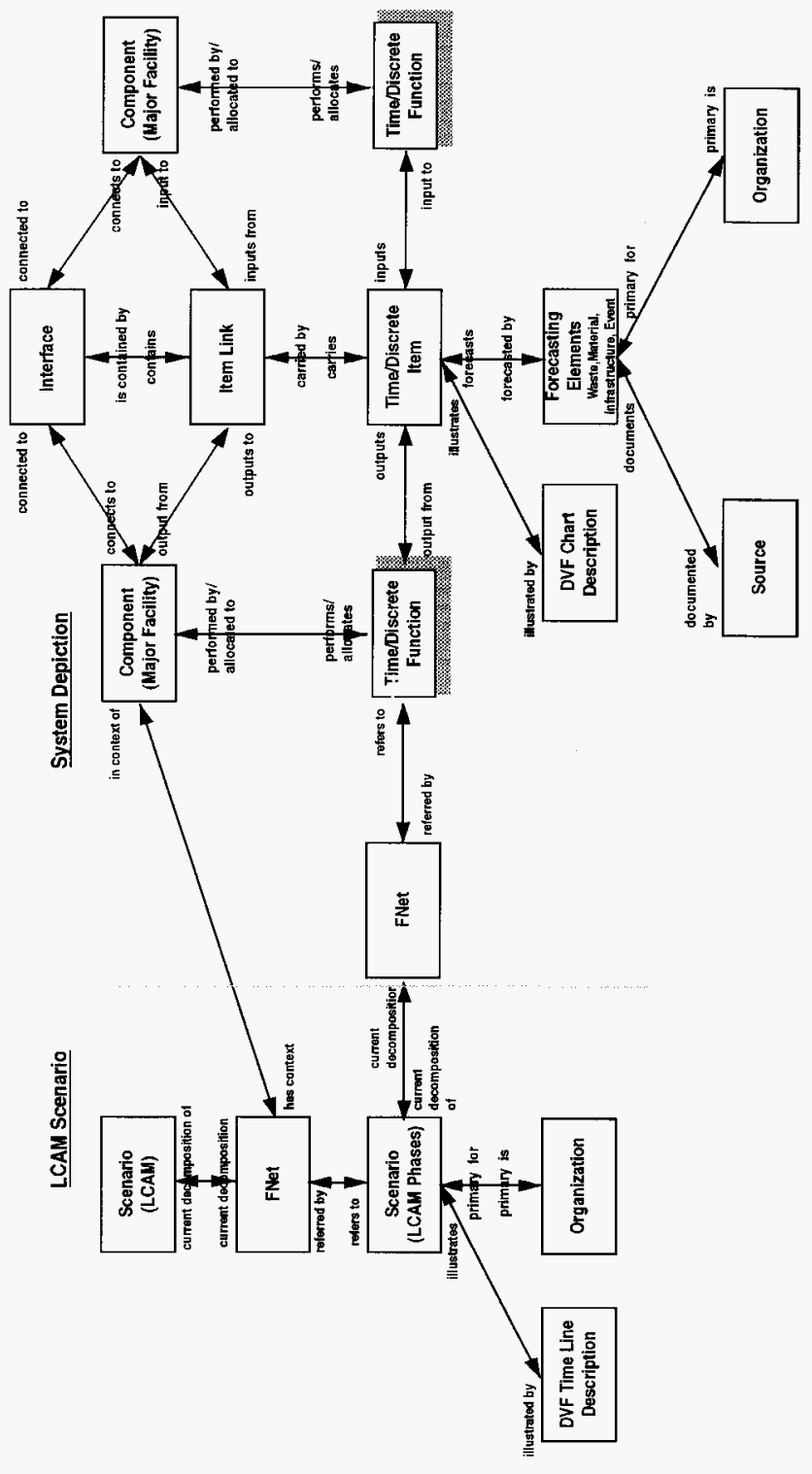




\section{Comments}

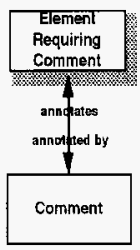

Categories

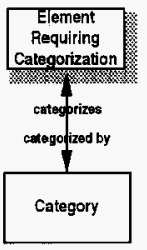

Requirements

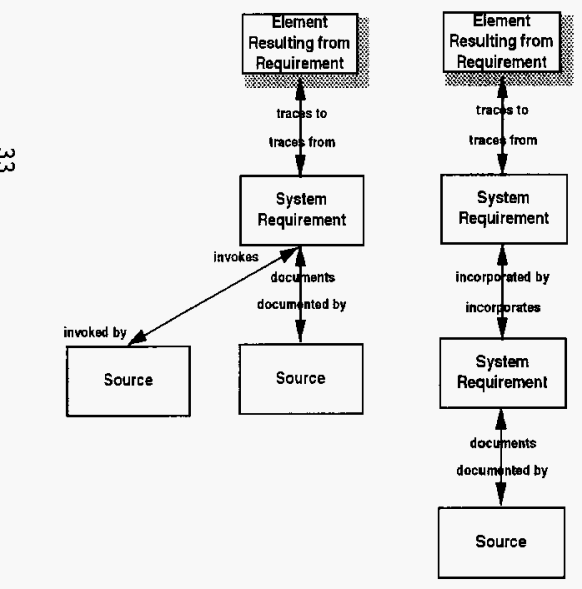

\section{Issue Tracking and Resolution}

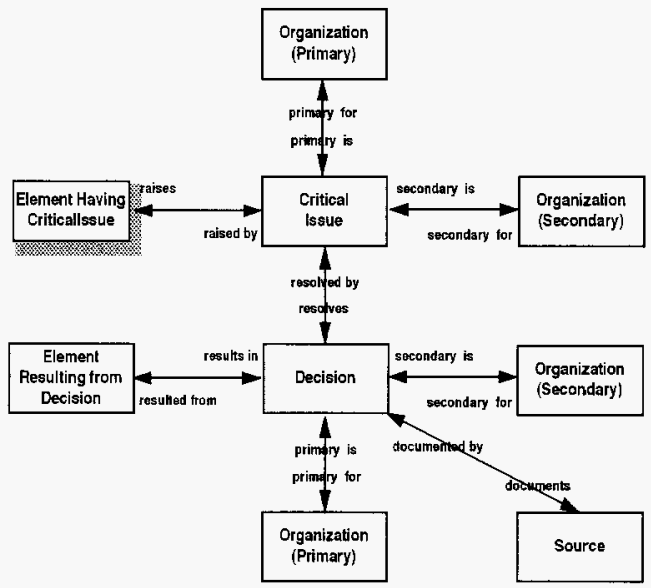

Hierarchical Relationships

Organizational Relationships

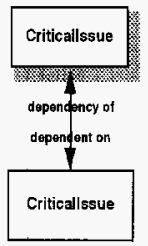

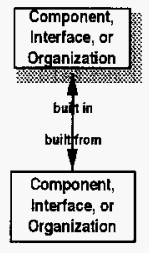

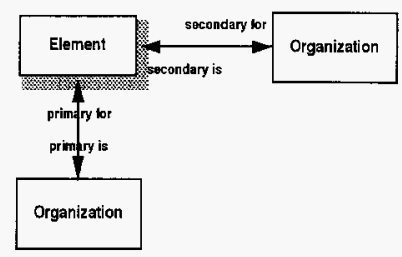


DISTRIBUTION SHEET

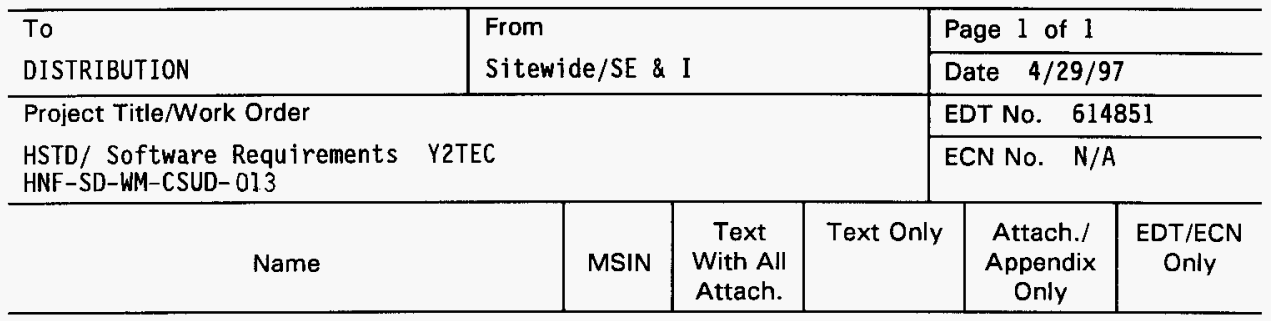

K. B. Bailey

S. S. Bath

P. A. Baynes

H8-71 $X$

H8-71 $X$

H8-71 $X$

J. E. Ferguson

H8-71 $X$

H. L. Garrison

H8-71 $X$

M. L. Grygiel

A. K. Lee

R. S. McBeath

T. E. Miller

D. J. Sommer

H8-71

H8-71

H8-71

H8-71

H8-71

$X$
$X$
$X$
$X$
$X$

$X$

$X$

$X$

$X$

Central Files

A3-88

$X$ 